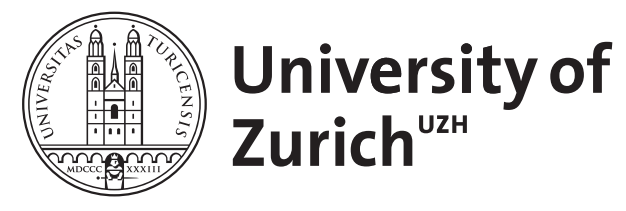

Zurich Open Repository and Archive

University of Zurich

University Library

Strickhofstrasse 39

CH-8057 Zurich

www.zora.uzh.ch

Year: 1996

Ultrafiltrationskontrolle mittels Hämatokritmessung bei der Hämodialyse

Polaschegg, Hans-Dietrich ; Knoflach, A ; Binswanger, U

DOI: https://doi.org/10.1515/bmte.1996.41.s1.374

Posted at the Zurich Open Repository and Archive, University of Zurich

ZORA URL: https://doi.org/10.5167/uzh-154360

Journal Article

Published Version

Originally published at:

Polaschegg, Hans-Dietrich; Knoflach, A; Binswanger, U (1996). Ultrafiltrationskontrolle mittels Hämatokritmessung bei der Hämodialyse. Biomedizinische Technik. Biomedical engineering, 41(s1):374-375.

DOI: https://doi.org/10.1515/bmte.1996.41.s1.374 


\title{
Ultrafiltrationskontrolle mittels Hämatokritmessung bei der Hämodialyse
}

\author{
Polaschegg Hans-Dietrich, Knoflach A.", Binswanger U." \\ Berater für Medizintechnik, Grünwiesenweg 9, D-61440 Obenursel, Deutschland \\ "Universitätsspital Zürich, Abt. Nephrologie, Ch-8091 Zürich, Schweiz
}

\section{EINLEITUNG:}

Bei Ausfall der Nieren sammelt sich Wasser im Körper an, das bei der Hämodialysebehandlung entzogen werden muß. Der Entzug von Wasser erfolgt durch Ultrafiltration (UF), wobei die zu entziehende Menge aus der Differenz zwischen dem Körpergewicht vor der Behandlung und einem nach klinischen Gesichtspunkten festgelegtem "Trockengewicht" erfolgt. Während der Hämodialyse kommt es, mit unterschiedlicher Häufigkeit, zu Symptomen wie Blutdruckabfällen, Krämpfen und Kopfschmerzen deren Ursachen primär im Flüssigkeitsentzug gesehen werden. In der Regel ist dafuir eine nicht individuell angepaßte UF-Rate oder ein unrichtiges Trockengewicht ${ }^{1}$ verantwortlich. Ein Versagen der UFKontrolleinrichtung kann ebenfalls zu einem überhöhten Flüssigkeitsentzug oder sogar zu einer gefährlichen Flüssigkeitsaufnahme des Patienten führen ${ }^{2}$.

Es stehen heute Monitore zur Verfügung, die die hauptsächlich UF-bedingte Veränderung des Blutvolumens (BV) kontinuierlich messen. Unter den anwendbaren Verfahren hat sich vor allem eines ausgezeichnet, das die Messung des absoluten Hämatokrits (Hkt) und nicht nur dessen relativer Veränderung erlaubt ${ }^{3}$. Damit stehen nun neue Möglichkeiten zur UF-Kontrolle zur Verfügung.

\section{SICHERHEITSFRAGEN ZUR ULTRAFIL TRATION:}

Die treibende Kraft für die UF ist ein, über die Membran zwischen Blut- und Dialysatraum des Dialysators wirkender, Transmembrandruck (TMP). Die UF-Rate hängt bei einem bestimmten TMP von Membran- und Bluteigenschaften ab und ist zeitlich nicht konstant. Bei hochpermeablen Membranen ist diese Empfindlichkeit besonders ausgeprägt.

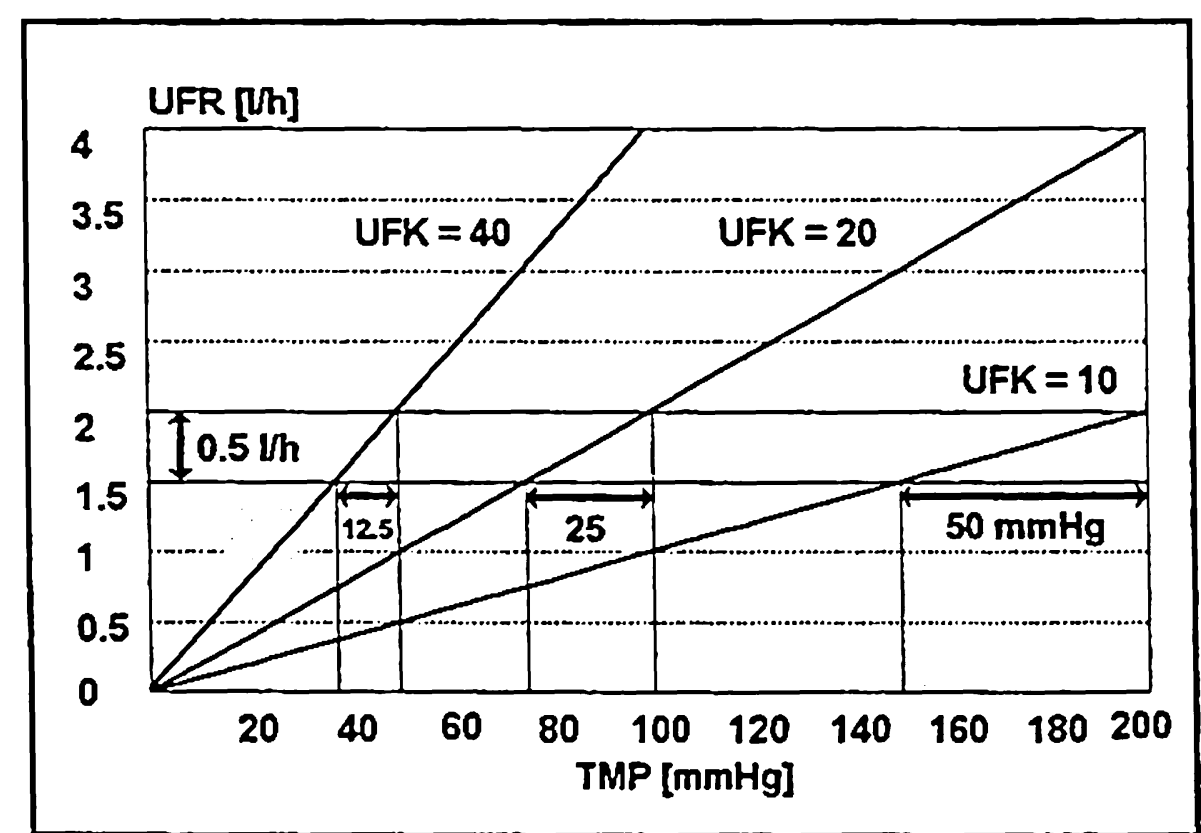

Abbildung 1: Die Auflösung der Transmembrandruckmonitore liegt bei $20 \mathrm{mmHg}$. Damit ist es nicht möglich, im Fehlerfall, bei Dialysatoren mit einem UF-

Koeffizienten von $>25 \mathrm{~m} / \mathrm{h} . \mathrm{mmHg}$ Abweichungen der Ultrafiltrationsrate von $0.5 \mathrm{l} / \mathrm{h}$ zu erkennen.
Deshalb werden für den Betrieb mit Dialysatoren mit solchen Membranen ausschließlich Geräte eingesettt, die Einrichtungen zur kontrollierten UF besitzen. Dem sicherheitstechnischen Standard entsprechend, müssen solche Geräte über ein Schutzsystem verfügen, das eine gefährliche Abweichung von der eingestellten UF-Rate im Fehlerfalle verhindert. Die Überwachung des TMP gilt als hinreichend. Für hochpermeable Membrane trifft dies jedoch nicht zu, wie Stragier et.al. (2) gezeigt haben. Wie aus Abb.1 hervorgeht, kann in diesem Fall die übliche TMP-Überwachung UF-Fehler von $0.5 \mathrm{l} / \mathrm{h}$ nicht erkennen.

\section{PHYSIOLOGIE DER WASSERSPEICHERUNG BEIM NIERENVERSAGEN:}

Im wesentlichen wird aufgenommenes Wasser im Extrazellularraum des Körpers gespeichert. Die Aufteilung erfolgt entsprechend einer, von Guyton angegebenen Funktion ${ }^{4}$. Danach wird Wasser zunächst zu 2/5 im vasculären System eingelagert. Bei Anstieg des BV von 51 (normal) auf 71 ist die Aufnahmefähigkeit des vasculären Systems erschöpft und es kommt zur Bildung von Ödemen. Bei langsamen Flüssigkeitsentzug kommt es gleichfalls zu einer Abnahme im Verhältnis 2:5 im vasculären System, wobei ein gesunder Organismus einen BV-Verlust bis 11 kompensieren kann. Bei Überschreitung bricht der Blutdruck zusammen. Bei schnellem Flüssigkeitsentzug nimmt das BV wesentlich stärker $a b$, da die Flüssigkeit aus dem interstitiellem Raum nur langsam nachströmt. Bei Unterbrechung des Flüssigkeitsentzugs wird deshalb ein Wiederanstieg des BV (rebound) beobachtet.

\section{SYMPTOME UND BLUTVOLUMENVERÄNDERUNG WÄHREND DER HÄMODIALYSE:}

Schon frühzeitig in der Entwicklung der Hämodialyse hat man versucht, BV-Veränderungen zur Vorhersage und Verhinderung von symptomatischen Blutdruckabfällen heranzuziehen. Kim und Mitarbeiters konnten keinen Zusammenhang zwischen relativen BV-Veränderungen und Blutdruckabfällen erkennen, stellten aber fest, daß Blutdruckabfälle immer dann auftraten, wenn das BV unter $50 \mathrm{ml} / \mathrm{kg}$ Körpergewicht abfiel. Dies ist bei Berücksichtigung der physiologischen Verhältnisse verständlich. Da die Flüssigkeitsaufnahme im interdialytischen Intervall unterschiedlich ist, wird auch die relative Veränderung des BV bis zum Erreichen des Trockengewichtes unterschiedlich sein. Da die Funktion BV-Veränderung gegen UF-Volumen überdies in individuell unterschiedlicher Weise nichtlinear ist, ist auch eine Vorherberechnung der idealen BV-Abnahme nicht möglich. Steuer et.al.(3) haben erkannt, daß Symptome auffällig häufig bei einem individuell unterschiedlichen, aber sonst gleichem Hktwert auftreten. Da ein Hhtwert einem bestimmten $\mathrm{BV}$ entspricht und sich die Zahl der roten 
Blutkörperchen von Dialyse zu Dialyse normalerweise nicht stark verändert, kann dieser Hhtwert ("Crash-Crit") zur Begrenzung der BV-Abnahme herangezogen werden. Dazu muß lediglich die UF bei Erreichen eines Schwellenwertes, der unterhalb des "Crash-Crit" liegt, unterbrochen werden. Mit einer manuellen Steuerung nach diesem Prinzip konnte eine wesentliche Verringerung intradialytischer Symptome beobachtet werden.

\section{BLUTVOLUMEN- UND HÄMATOKRITSENSOREN:}

Das BV wird aus der Konzentration einer Markersubstanz, deren Menge bekannt ist, bestimmt. Als Markersubstanz werden z.B. radioaktiv markierte Erythrozyten verwendet. Für die klinische Anwendung ist dieses Verfahren ungeeignet. Man verwendet deshalb die natürlich vorhandenen Erythrozyten oder das Gesamtblutprotein als Markierungssubstanz. Da die absolute Menge nicht bekannt ist, läßt sich lediglich die relative Veränderung bestimmen:

$$
\Delta B V=\frac{H k t_{\text {start }}}{H k t_{\text {ist }}}-1
$$

Die Messung des Hhts oder eines ihm proportionalen Wertes karm mit Hilfe unterschiedlicher Verfahren erfolgen, die alle Nebenempfindlichkeiten gegenüber anderen Parametern aufweisen. Bei optischen Verfahren dominiert die Streuung an Erythrozyten. Die Streueffekte, die vom Protein- und Elektrolytgehalt abhängen, können durch Messung bei zwei Wellenlänge kompensiert werde. Der Crit-Line-Monitor von In-Line-Diagnostics, Riverdale, Utah, USA setzt dieses Verfahren ein. Dieses Gerät erlaubt mit Hilfe eines speziellen Einmalartikels im Blutschlauchsystem die kontinuierliche Registrierung des Hkt mit einer Genauigkeit von $\pm 1 \%$. Bei Überschreiten einer Hht-Alarmschwelle erzeugt das Gerät

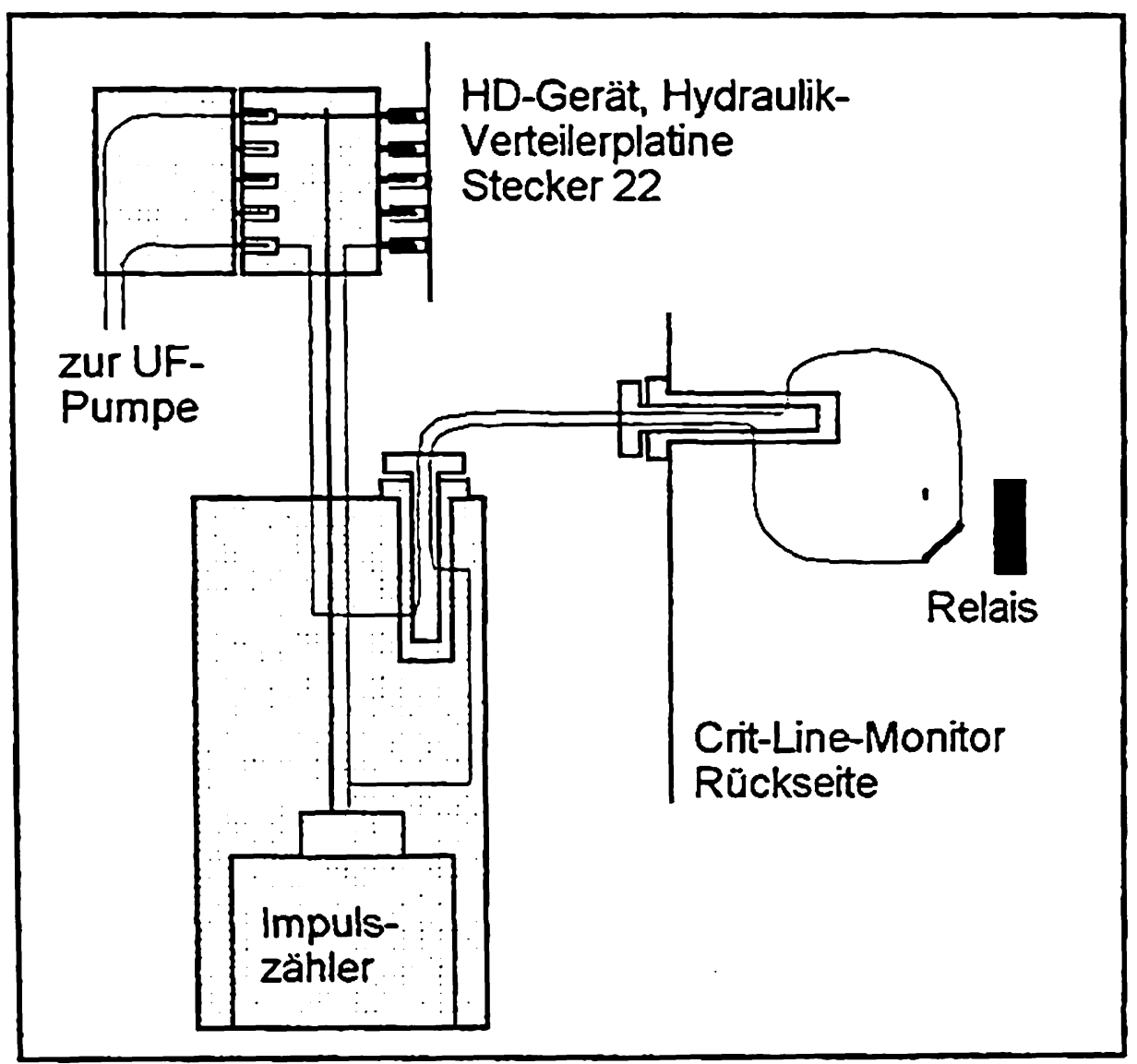

Abbildung 2: Adapterschaltung.In Ruhestellung ist das Relais geschlossen und Steuerimpulse gelangen von der Verteilerplatine des Dialysegerätes zur UF-Pumpe und zum Impulszähler. Im Alarmfall schaltet das Relais und die Verbindung zur UF-Pumpe und dem Impulszähler wird unterbrochen. einen optischen und akustischen Alarm und schaltet gleichzeitig ein Relais um.

\section{DER ULTRAFILTRATIONSKONTROLLADAPTER:}

Für die Hämodialysegeräte $2008 \mathrm{C}$ und 2008E der Fresenius AG, Oberursel wurde ein Adapter entwickelt, der bei Überschreiten des Alarmgrenzwertes des Crit-Line-Monitors die UF-Pumpe abschaltet und damit die UF unterbricht. Sinkt der Hkt durch "refilling" wieder unter die Alarmschwelle, so wird die UF entsprechend der, an der Maschine vorgegebenen Rate, weitergeführt. Da während der Unterbrechung der UF der im Dialysegerät eingebaute UF-Zähler weiterläuft, enthält der Adapter einen unabhängigen Zähler, der das tatsächliche UF-Volumen anzeigt, wogegen der Zähler des Dialysegerätes das Soll-UF-Volumen registriert.

\section{KLINISCHE ERPROBUNG:}

Ein Patient, bei dem bei vorhergegangenen Behandlungen Blutdruckabfalle auftraten, wurde mit dem Verfahren behandelt, wobei der "Crash-Crit" aus den vorherigen Behandlungen übernommen wurde. Dadurch wurde ein Blutdruckabfall vermieden. Den Behandlungsverlauf zeigt Abbildung 3: Mittlerweilen wurde diese Behandlungsmethode erfolgreich bei sieben weiteren Patienten eingesetzt $t^{6}$.

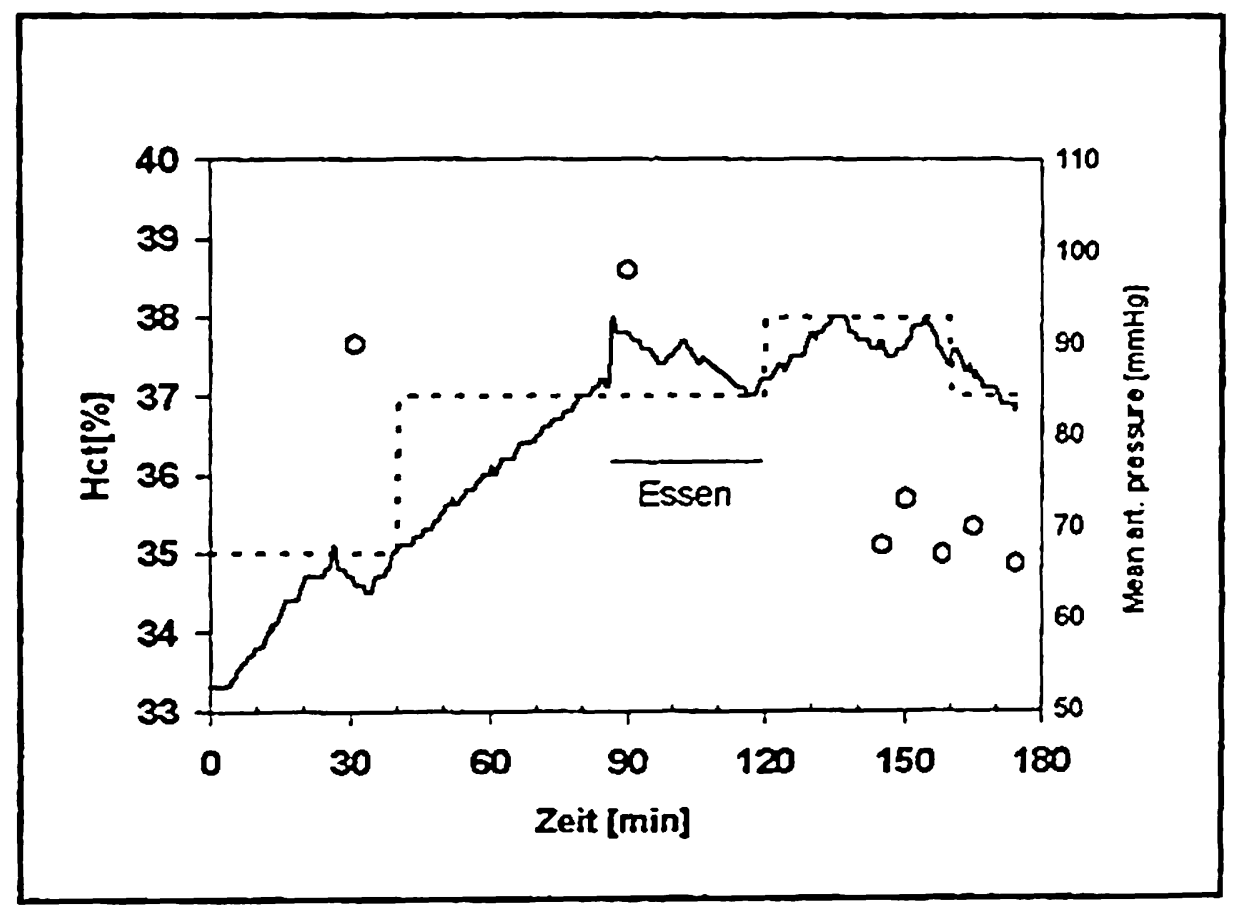

Abbildung 3: Volle Linie: Hkt, Kreise: Mittlerer, arterieller Blutdruck. Strichlierte Linie: HktAlarmgrenzwert. Dieser wurde zunächst zur Funktionsprüfung auf $35 \%$ gesetzt, danach auf $1 \%$ unter den Crash-Crit und nach dem Essen auf 38\%. Der Blutdruck fiel dabei unsymptomatisch $a b$.

\section{LITERATUR:}

1.Techert $F$ et.al. EDTNA-ERCA Journal 1993;19:5-6

2.Stragier A et.al. EDTNA-ERCA Journal 1996;22:35

3.Steuer RR et.al. ASAIO Journal 1994;40:M691-6

4.Guyton AC. Textbook of Medical Physiology, Eight Edition. W.B.Saunders Company, 1991

5.Kim KE et.al. ASAIO Transactions 1970;16:508-14 6.Knoflach A et.al. Abstract ASN 1996 